The researchers said that electrical contact was established the instant the nanotube was soldered to the second electrode. Metallic conduction was indicated by a linear current-voltage relationship. The researchers connected four nanotubes to microelectrode pairs and obtained reliable ohmic contacts with resistances in the range of 9-29 $\mathrm{k} \Omega$. The resistances show no clear correlation to the lengths of the bridges, were unaffected by the nanotube extensions, and were found to be constant in air for several days.

The researchers verified the intrinsic conductivity of the soldering material by depositing gold-carbon bridges between microelectrode pairs and measuring the current-voltage characteristics. Ohmic resistances between $80 \mathrm{k} \Omega$ and $520 \mathrm{k} \Omega$ were observed. By using scanning electron microscopic images to estimate the bridge's cross section and assuming a $60-\Omega$ serial resistance, the researchers calculated resistivities of $\sim 10^{-4} \Omega \mathrm{cm}$. TEM analysis revealed that the soldering material is composed of a gold-carbon composite structure with a porous crust of 3-5 nanoparticles surrounding a dense core.

"There are strong indications that this core is almost pure gold," said Bøggild, "and we know now how to control the core diameter with respect to the crust to engineer the composition of the material.

Attaching multiwalled CNTs to microelectrodes with a nonmetallic carbonaceous material resulted in devices with electrical conduction in the megohm range, which the researchers believe indicates that the metallic content of the soldering material is necessary for good electrical contact.

The researchers found the soldering bonds to be mechanically strong compared to the multiwalled CNTs. Further quantitative investigation will involve integration of a piezoresistive force sensor into their setup. The researchers said that they "anticipate automated electron-beam nanosoldering to be useful for quickly connecting complex circuitry consisting of nanoscale components in a way similar to the soldering of electronic components on the macroscale."

Bøggild added, "The solder material is a metal-containing gas, the soldering iron is a beam of electrons, but apart from that, it's basically the same thing."

STEVEN TROHALAKI

\section{Electrochemical Technique Forms High Corrosion-Resistant Silicate Layer on Zn-Plated Steel}

Currently, Cr conversion coatings, which are deposited from toxic hexavalent baths, are used to protect steel, zinc, and other metals from corrosion, but silicate coatings are being considered as a substitute. The silica deposit yields better corrosion characteristics as compared to $\mathrm{Cr}$ conversion coatings, but the fabrication procedure is laborious, time-consuming, and not suitable for commercial application. As reported in the February issue of Electrochemical and Solid-State Letters, a group of researchers from the University of South Carolina and Elisha Technologies Co. have synthesized a stable deposit with uniform layers of $\mathrm{SiO}_{2}$ on a zinc surface by cathodic electrolysis of zinc-plated panels.

B.N. Popov from the university and coworkers used an $\mathrm{N}$ sodium silicate solution with $28.4 \mathrm{wt} \% \mathrm{SiO}_{2}, 9.1 \mathrm{wt} \% \mathrm{Na}_{2} \mathrm{O}$, and $62.5 \mathrm{wt} \% \mathrm{H}_{2} \mathrm{O}$ for $\mathrm{SiO}_{2}$ electrodeposition. They found that optimal conditions for cathodic electrodeposition of a dense $\mathrm{SiO}_{2}$ film on $\mathrm{Zn}$-coated steel consist of a concentration of $5.6 \mathrm{wt} \%$ of sodium silicate in water $(\mathrm{pH}=10.5)$, potential $12 \mathrm{~V}$ (in a two-electrode cell) for $15 \mathrm{~min}$ at $75^{\circ} \mathrm{C}$. A $\mathrm{SiO}_{2}$ film forms because of an increase in $\mathrm{pH}$ near the electrode surface and hence, the polymerization of silicates on the surface. At the same time, dipping the same sample into the solution with no current applied did not lead to the formation of $\mathrm{SiO}_{2}$ film. Air-drying the samples after electrodeposition significantly increased the resistance of the coatings due to a decrease in the average crack size in the coating, as confirmed by scanning electron microscopy. Corrosion resistance measurements showed that the silicate coating provided a much better performance with at least one order of magnitude higher resistance than the $\mathrm{Cr}$ conversion coating.

MAXIM NIKIFOROV

\section{Ellipsometry Achieves Determination of Optical Constants and Crystal Orientation for Biaxial Absorbing Materials}

Ellipsometry is a well-known technique used to determine optical constants from isotropic bulk and thin-film structures, and generalized ellipsometry extends to layered anisotropic materials. In the December 1, 2002 issue of Optics Letters, M. Schubert (Universität Leipzig, Germany and University of Lincoln-Nebraska, USA) and W. Dollase (University of California, Los Angeles, USA and Universität Bayreuth, Germany) have shown that the generalized ellipsometry technique is able to determine not only all optical constants of a biaxial absorbing material, meaning the indices of refraction and extinction coefficients for the three principal axes of refraction, but also the specific crystal orientation from a single bulk sample.

The researchers studied the metal chalcogenide stibnite $\left(\mathrm{Sb}_{2} \mathrm{~S}_{3}\right)$, an ortho- rhombic material that has become of interest recently because of its use in television cameras, microwave switching, and optoelectronic devices. Ellipsometric measurements were performed for a wavelength of $589 \mathrm{~nm}$, for incidence angles from $20^{\circ}$ to $70^{\circ}$, every $10^{\circ}$, and as a function of the in-plane sample rotation, that is, for a full sample azimuth revolution. The technique revealed the three principal indices of refraction (3.379, $5.075,4.417)$ and the three extinction coefficients (0.090, 0.1, 0.27). Furthermore, assuming the existence of an oxide overlayer on the surface of the material, the data analysis showed an index of refraction of 1.15 , and a thickness of the order of 11-12 $\mathrm{nm}$. The absolute orientation of the samples was also determined through the identification of the three Euler angles that describe the orientation of the crystal axes relative to the lab axes. The orientation for three different samples reached excellent agreement with that obtained by $x$-ray diffraction. The researchers suggest that generalized ellipsometry could be a powerful tool for measurement of anisotropic optical function spectra of biaxial materials.

ROSALIA SERNA

\section{High-Efficiency Electro-Optic Modulator with Low-Loss Waveguides Fabricated}

Electro-optic (EO) waveguides and modulators are critical components in modern fiber-optic communications systems. Currently produced devices are limited by high loss of light signal in the nearIR region. Recently, the fabrication and testing of a potentially superior EO modulator design was reported by W.H. Steier and co-workers in the Department of Electrical Engineering at the University of Southern California along with researchers at Zen Photonics in Daejon, Korea, and Pacific Wave Industries in Los Angeles, Calif. The researchers describe a MachZehnder (MZ) modulator which incorporates low loss passive polymers in noncritical areas of the device, and features passive polymer waveguides to reduce signal loss due to mode mismatch between the modulator and connecting optical fibers. While the created device exhibited lower efficiency than their best modulators, the researchers believe that this reflects a limitation of the materials used for device construction and not the design itself. According to Steier, "The integration of passive and EO polymers makes this a practical approach for use in large-scale optical integrated circuits."

As described in the December 1, 2002, issue of Optics Letters, the researchers used 
fluorinated resins ZPU-1279 and ZPU-1215 for the cladding and passive core regions, respectively. The EO (active) polymer was a guest-host complex of CLD-1 chromophore and amorphous polycarbonate (APC). The device was constructed by spincoating the lower cladding and the passive core layers of the device onto a silicon substrate. A layer of the EO polymer was then deposited on top of the passive layer, the nonessential areas removed by reactive ion etching, and vertical tapers etched to reduce the loss as the light couples from the passive to the $\mathrm{EO}$ waveguides. The active structure was then covered with the upper passive core and cladding layers. Standard photolithography was used to create the waveguide patterns and gold electrodes were formed by vacuum evaporation and photolithography inside both cladding layers. The arms of the modulator were poled, that is, the CLD-1 chromophores were aligned, by application of $500 \mathrm{~V}$ poling voltage with the modulator held at $145^{\circ} \mathrm{C}$ in $\mathrm{N}_{2}$ atmosphere.

The modulator efficiency was measured by coupling $1.55-\mu \mathrm{m}$ transverse-magneticpolarized light into the device and focusing the output signal onto a photodetector. The measured EO coefficient was $20 \%$ smaller than the best values exhibited by the researchers' conventional modulators utilizing the same EO polymer. The researchers believe that the low efficiency is the result of incomplete poling due to the high resistivity of the passive core polymer, and will improve if different materials are used. Mode profile studies, on the other hand, showed a tenfold reduction in fiber-waveguide coupling loss and a reduced chip loss when an EO polymer was replaced by the passive material used in this study.

GREG KHITROV

\section{Inert Liquid Paraffin Speeds Preparation of Large, Crack-Free Mesostructured Silica Monoliths}

Bulk mesostructured materials with a high orientation order at the macrolevel are attracting current interest, in particular for developing novel sensors, micro- and optoelectronics, and separation devices. However, industrial applications are limited because of the absence of an adequate route for the preparation of large-sized, crack-free silica monoliths. Researchers from the Department of Chemistry of Fudan University, Shanghai, China, have demonstrated a fast way to prepare mesoporous silica monoliths by using solvent evaporation and a liquid paraffin medium as a morphology protector, as reported in the January issue of Chemistry of Materials.

Dongyuan Zhao and co-workers synthesized mesostructured silica monoliths through solvent evaporation from the silica gel by using tetraethoxysilane as a silica source and poly-(ethylene oxide)-poly (propylene oxide)-poly(ethylene oxide) $\left(\mathrm{EO}_{20} \mathrm{PO}_{70} \mathrm{EO}_{20}\right)$ amphiphilic block copolymer as a template. They removed

\section{Space Shuttle Columbia Disaster: Materials Issues www.mrs.org/gateway/columbia.html}

While returning from orbit on February 1, 2003, the Space Shuttle Columbia and all seven crewmembers were lost over north central Texas. Columbia was returning from a 16-day scientific research mission. Aboard Columbia were Commander Rick Husband; Pilot William McCool; Mission Specialists Dave Brown, Kalpana Chawla, and Laurel Clark; Payload Commander Mike Anderson; and Payload Specialist Ilan Ramon of the Israel Space Agency.

Initial speculation suggested that materials-related failure issues may have contributed to the shuttle accident. However, at press time, the conclusion was far from certain.

NASA Administrator Sean O'Keefe announced the formation of a Space Shuttle Mishap Interagency Investigation Board, which will provide an independent review of the events and activities that led up to the Columbia tragedy. The board is chaired by retired U.S. Navy Admiral Harold W. Gehman Jr.

The research mission, identified as STS-107, included over 80 experiments. Among the materials-related studies was one on zeolite crystal growth (Center for Advanced Microgravity Materials Processing, Northeastern University, Boston). The purpose of this continuing study is to understand how to manipulate the nucleation and growth of zeolite in order to use it for hydrogen-fuel storage. In another experiment, students from Ort-Matzkin School (Haifa, Israel) participating in NASA's educational program sent a chemical garden in which the growth mechanisms of crystalline fibers in microgravity were investigated.

For updates on materials news relating to the Columbia disaster, access the Materials Research Society Web site at www.mrs.org/gateway/columbia.html.

the residue of the solvent by heating the obtained silica gel, covered by a layer of inert liquid paraffin, at relatively high temperatures $\left(60-90^{\circ} \mathrm{C}\right)$, which reduced the processing period to $8 \mathrm{~h}$ and preserved the macromorphology of the monolith from cracking.

According to the theory of the sol-gel process, the stress that causes cracking is mainly attributed to the internal pressure gradient, which arises as a result of the loss of permeability of the gel or different diameters of the pores. Liquid paraffin is able to fully separate the gel from the atmosphere, reducing damage caused by the internal pressure gradient in three ways. The evaporation rate during heating accelerates slowly and the viscosity of the liquid decreases, which increases the permeability of the gel. The flaws at the surface of the gel are filled with paraffin, replacing the initial alcohol-vapor interface by the alcohol-paraffin interface. This reduces the internal pressure that would otherwise pull the flaws open. During heating, paraffin restrains the rates of evaporation in the pores of all sizes as compared to those without a paraffin cover, which keeps the pores empty for the most part. This technique, the researchers conclude, prevents the gel from cracking while attaining uniform shrinkage. Furthermore, Fourier transform infrared spectroscopy revealed that paraffin does not penetrate into the material, thus the paraffin acts only as a macromorphology protector, and does not change the chemical composition of the final mesostructured silica monolith.

ANDREI A. ELISEEV

\section{FOR MORE RESEARCI NEWS ON MATERIALS SCIENCE . . \\ .. access the Materials Research Society Web site: \\ www.mrs.org/gateway/ matl_news.html}

\section{Correction}

In the February 2003 issue of MRS Bulletin, page 143, the correct affiliation for Stanley Israel (far right in the photo) is a Member of the Board of the American Chemical Society.
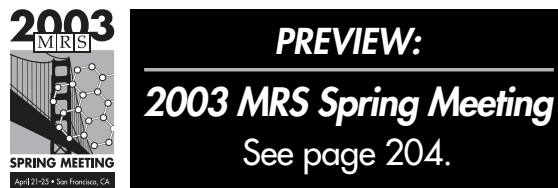